\title{
Absence of the Kasner singularity in the effective dynamics from loop quantum cosmology
}

\author{
Ghanashyam Date* \\ The Institute of Mathematical Sciences, CIT Campus, Chennai-600 113, INDIA
}

\begin{abstract}
In classical general relativity, the generic approach to the initial singularity is usually understood in terms of the BKL scenario. In this scenario, along with the Bianchi IX model, the exact, singular, Kasner solution of vacuum Bianchi I model also plays a pivotal role. Using an effective classical Hamiltonian obtained from loop quantization of vacuum Bianchi I model, exact solution is obtained which is non-singular due to a discreteness parameter. The solution is parameterized in exactly the same manner as the usual Kasner solution and reduces to the Kasner solution as discreteness parameter is taken to zero. At the effective Hamiltonian level, the avoidance of Kasner singularity uses a mechanism distinct from the 'inverse volume' modifications characteristic of loop quantum cosmology.
\end{abstract}

PACS numbers: 04.60.Pp,98.80.Jk,98.80.Bp

While the celebrated singularity theorems of classical general relativity imply that the backward evolution of an expanding universe leads to a singular state, the nature of the singularity is elucidated in terms of the BelinskiiKhalatnikov-Lifschitz (BKL) scenario [1, 2]. The scenario views the spatial slice close to the (space-like) singularity as made up of approximately homogeneous patches each of which evolves according to the vacuum Bianchi IX model. As the singularity is approached, the patches fragment indefinitely, asymptotically becoming infinitely small. The vacuum Bianchi IX evolution in turn, can be viewed as a succession of Bianchi I evolutions (Kasner epochs), interleaved by transitions among the Kasner epochs. This interleaved evolution continues indefinitely in a chaotic manner. Apart from the indefinite number of Kasner epochs and transitions among these, the monotonic decrease of the volume and the singular nature of the Kasner solution are responsible for the infinite fragmentation of the homogeneous patches.

Singularities 'evolving' from non-singular physical situations are indicative of breakdown of the extrapolation of the classical evolution i.e. dynamics of Einstein equation and call far an extension/modification of the classical theory/framework. One natural avenue is to appeal to a corresponding quantum theory of gravity. Since the classical picture of the singular behaviour involves highly dynamical geometries with arbitrarily large curvatures, a quantum theory which does not depend on any preselected background geometry is likely to be most suitable for obtaining the required extensions.

Loop quantum gravity (LQG) is precisely such a background independent approach [3]. While the LQG for the general inhomogeneous situations is quite complicated, its methods can be implemented and tested in simpler contexts of spatially homogeneous geometries. Indeed, loop quantization of the so-called diagonalized, Bianchi class A models has been carried out [4, 5] and shown to be non-singular within the quantum framework. In the quantum framework, non-singularity means non-breakdown of the fundamental dynamical equations (which are partial difference equations) and boundedness of relevant operators such as the inverse triad operator which enter the quantization of curvature components, matter densities etc.

It is of course more intuitive and convenient to obtain the modifications implied by the quantum theory in the familiar geometrical setting of classical general relativity i.e. obtaining the modifications to the Einstein dynamics keeping the kinematical framework of Riemannian geometry in tact. This has been done systematically for the isotropic models [ $[$, , 7, , 8] in terms of an effective Hamiltonian. The derivation of the effective Hamiltonian is based on the observation that if the fundamental dynamical equations admits a solution which is WKB approximable (i.e. the amplitude and the phase are slowly varying in a suitable sense) in some domain, then to the order $\hbar^{0}$ one obtains a Hamilton-Jacobi equation from which an effective classical Hamiltonian can be read-off, also valid within the same domain. The largest possible domain of validity of such an approximation is constrained by the classical 'turning points' dictated by the effective Hamiltonian. The form of the effective Hamiltonian so obtained does not depend on details of the presumed solution, but of course the actual domain of validity of the WKB approximation and consequently of the effective Hamiltonian is sensitive to the presumed solution. Being $o\left(\hbar^{0}\right)$, the effective Hamiltonian is insensitive to factor ordering issues.

There are various types of corrections that arise. The most dramatic one is the correction implied by the nontrivial quantization of inverses of various classical quantities such as scale factors, triad components, volume etc [9, 10]. In the isotropic context, this has lead to a variety of implications [11, 12, 13, 14]. For the anisotropic context, this leads to suppression of chaotic behaviour of the Bianchi IX model [15] with the further result that asymptotically for vanishing volumes, a Bianchi IX solution approaches a Kasner solution in a stable manner 
[16].

For the vacuum Bianchi I model, there is no potential term and no scope for a modification of dynamics due to quantizations of inverses of triad components. Although at the quantum level, there is no singularity [4], the effective classical Hamiltonian derived from a continuum approximation, is identical to the Einstein Hamiltonian leading to the same singular Kasner solution. Recently however an alternative method of deriving effective classical Hamiltonian has been devised, in the context of isotropic models [8], which uses the WKB ansatz directly at the difference equation level bypassing the step of first deriving the Wheeler-De Witt differential equation to be followed by WKB approximation. The same method can also be applied in the anisotropic context which leads to an effective Hamiltonian different from the Einstein Hamiltonian. Exact, non-singular solution of this Hamiltonian for the vacuum Bianchi I model is the result presented here.

A Bianchi I space-time is specified in terms of the metric of the form,

$$
d s^{2}=d t^{2}-\sum_{I} a_{I}^{2}(t)\left(d x^{I}\right)^{2},
$$

where $t$ is the synchronous time. The vacuum Einstein equations then lead to the well known Kasner solution: $a_{I}(t) \sim t^{2 \alpha_{I}}$ where $\alpha_{I}$ are constants satisfying the conditions $\sum_{I} \alpha_{I}^{2}=1=\sum_{I} \alpha_{I}$. For subsequent comparison with the new solution, it is convenient to describe the time evolution in terms of a new time coordinate $\tau$ corresponding to the lapse $\mathcal{N}:=a_{1} a_{2} a_{3}$, defined by $\mathcal{N} d \tau=d t$. The scale factors then evolve as $a_{I} \sim e^{\alpha_{I} \tau}$.

Loop quantization of all diagonalized, Bianchi class A models has been given in [5]. Briefly, it may be summarized as follows. The kinematical Hilbert space is spanned by orthonormalized vectors labeled as $\left|\mu_{1}, \mu_{2}, \mu_{3}\right\rangle, \mu_{I} \in \mathbb{R}$. These are properly normalized eigenvectors of the triad operators $p^{I}$ with eigenvalues $\frac{1}{2} \gamma \ell_{\mathrm{P}}^{2} \mu_{I}$, where $\gamma$ is the Barbero-Immirzi parameter and $\ell_{\mathrm{P}}^{2}:=8 \pi G \hbar:=\kappa \hbar$. The volume operator is also diagonal in these labels with eigenvalues $V(\vec{\mu})$ given by $\left(\frac{1}{2} \gamma \ell_{\mathrm{P}}^{2}\right)^{3 / 2} \sqrt{\left|\mu_{1} \mu_{2} \mu_{3}\right|}$. Here we have used the vector notation to denote the triple $\left(\mu_{1}, \mu_{2}, \mu_{3}\right)$. Imposing the Hamiltonian constraint operator on general vectors of the form $|s\rangle=\sum_{\vec{\mu}} s(\vec{\mu})|\vec{\mu}\rangle$ leads to the fundamental difference equation for the coefficients $s(\vec{\mu})$. Here the sum is over countable subsets of $\mathbb{R}^{3}$. There are further gauge invariance conditions [4] which do not concern us here.

In the present context of vacuum Bianchi I model, the fundamental difference equation takes the form [5],

$$
\sum_{\vec{\epsilon}_{12}} A_{12}\left(\vec{\mu} ; \vec{\epsilon}_{12}\right) s\left(\vec{\mu} ; \vec{\epsilon}_{12}\right)+\text { cyclic }=0, \text { where }
$$

$\vec{\epsilon}_{12}=\left(\epsilon_{1}, \epsilon_{2}, \epsilon_{1}^{\prime}, \epsilon_{2}^{\prime}\right)$ with each of the $\epsilon_{*}$ taking values \pm 1 ; $s\left(\vec{\mu} ; \vec{\epsilon}_{12}\right)=s\left(\mu_{1}-\mu_{0} \epsilon_{1}-\mu_{0} \epsilon_{1}^{\prime}, \mu_{2}-\mu_{0} \epsilon_{2}-\mu_{0} \epsilon_{2}^{\prime}, \mu_{2}\right) ; \mu_{0}$ is an order 1 parameter and,

$$
\begin{aligned}
A_{12}\left(\vec{\mu} ; \vec{\epsilon}_{12}\right) & =V\left(\vec{\mu} ; \vec{\epsilon}_{12}\right) d\left(\mu_{3}\right)\left(\epsilon_{1} \epsilon_{2}+\epsilon_{1}^{\prime} \epsilon_{2}^{\prime}\right) \\
d(\mu) & :=\left\{\begin{array}{cc}
\sqrt{\left|1+\mu_{0} \mu^{-1}\right|}-\sqrt{\left|1-\mu_{0} \mu^{-1}\right|} & \mu \neq 0 \\
0 & \mu=0
\end{array}\right.
\end{aligned}
$$

In the summary above, we have used the non-separable kinematical Hilbert space and also made the parameter $\mu_{0}$ explicit [17.

To derive the effective Hamiltonian, one assumes that there exist solution(s) of the partial difference equation which have a slowly varying amplitude and phase at least in some region of large volume. Explicitly, defining $\vec{p}\left(\mu_{I}\right):=\frac{1}{2} \gamma \ell_{\mathrm{P}}^{2} \vec{\mu}$, one introduces an interpolating function, $\psi(p)$, such that $s(\vec{\mu})=\psi\left(\vec{p}\left(\mu_{I}\right)\right)=C(\vec{p})$ $\exp \left\{\frac{i}{\hbar} \Phi(\vec{p})\right\}$ and assumes that the amplitude and phase are slowly varying functions of $\vec{p}$ in the sense that higher order terms in the Taylor series about any $\vec{p}$ in the relevant region, are smaller than the lower order terms when compared over the quantum geometry scale $q:=$ $\frac{1}{2} \gamma \mu_{0} \ell_{\mathrm{P}}^{2}$. Taylor expanding the interpolating wave function $\psi\left(\vec{p} ; \vec{\epsilon}_{I J}\right),(I J)=(12,23,31)$, it is straightforward to check that the leading terms (in powers of $\hbar$ ) in the real part of the equation are $o\left(\hbar^{0}\right)$ while those in the imaginary part are $o(\hbar)$. The $o\left(\hbar^{0}\right)$ terms involve only the first order partial derivatives of the WKB phase. Identifying $K_{1}:=\kappa \Phi_{1,0,0}(\vec{p})$ etc., one infers the Hamiltonian system from the Hamilton-Jacobi equation as: $\left\{p^{I}, K_{J}\right\}=\kappa \delta_{J}^{I}$, with the Hamiltonian $\left(\epsilon:=\mu_{0} \gamma\right)$,

$$
\kappa \mathcal{N} H_{\mathrm{eff}}(\vec{p}, \vec{K})=-2\left[p^{1} p^{2} \frac{\sin \epsilon K_{1}}{\epsilon} \frac{\sin \epsilon K_{2}}{\epsilon}+\operatorname{cyclic}\right](4)
$$

$\epsilon$ will be referred to as the discreteness parameter.

The effective Hamiltonian is periodic in $\epsilon K_{I}$ and we may restrict our attention to $-\pi<\epsilon K_{I}<\pi$. The imaginary part of the equation however requires the domain of validity to be restricted further in order to be self consistent with the assumption of slow variation of the interpolating wave function. The restriction is: $p_{I} \geq q$ and small neighbourhoods of $\pm \frac{\pi}{2}$ are to be excluded. Thus along the $\epsilon K_{I}$ axes, the effective Hamiltonian is a good approximation in the intervals: $\left(-\pi,-\frac{\pi}{2}-\delta\right),\left(-\frac{\pi}{2}+\delta, \frac{\pi}{2}-\delta\right),\left(\frac{\pi}{2}+\delta, \pi\right)$ for some small positive $\delta$. The values $\epsilon K_{I}= \pm \frac{\pi}{2}$ will turn out to be the 'turning points' of the trajectories, $\vec{p}(\tau)$, of the effective dynamics.

For $\epsilon K_{I} \sim 0$, one can use $\epsilon^{-1} \sin \epsilon K_{I} \approx K_{I}$. Then the effective Hamiltonian goes over to the Einstein Hamiltonian and the dependence on $\epsilon$ drops out. Keeping only the $\hbar^{0}$ terms, we have effectively taken $\left|p^{I}\right|$ larger than the quantum geometry scale set by $q$. Thus it is clear that Einstein dynamics is reproduced for large values of triads and small values of their conjugates $K_{I}$. In this regime, the Hamilton's equations for $p^{I}$, combined with the relation of the triad components to scale factors, $\left|p^{I}\right|=a_{J} a_{K}$ (and cyclic permutations), identifies the $K_{I}$ 's as components of the extrinsic curvature of the constant $t$ slices: 
$K_{I}=-\frac{1}{2} \frac{d a_{I}}{d t}$. The effective Hamiltonian thus deviates from the Einstein Hamiltonian mainly in the region of the phase space with not too small values of $\epsilon K_{I}$.

The small value of $\epsilon K_{I}$ can be achieved by taking the limit $\epsilon \rightarrow 0$ ( $K_{I}$ fixed) which then removes any restriction on $K_{I}$ 's. From a purely classical perspective, such a view may be welcome especially since the parameters $\mu_{0}, \gamma$ disappear which are absent in the Einstein dynamics. However, from a quantum perspective, the discreteness parameter cannot be zero [17]. For a non-zero value of discreteness parameter, the effective Hamiltonian represents an extension of Einstein dynamics for phase space regions beyond small extrinsic curvatures and large triad components. Since the non-zero value of $\epsilon$ reflects a discrete structure in a specific technical sense, the effective dynamics is to be viewed as a way of extending the Einstein dynamics by incorporating the discrete feature of quantum geometry.

We use geometrized units $(\kappa=1=c)$ so that all quantities have dimensions of powers of length. The triad variables $p^{I}$ and $\hbar$ have dimensions of (length) ${ }^{2}$; the effective Hamiltonian, scale factors, the synchronous time all have dimensions of length while the $\tau$ has dimensions of (length $)^{-2}$. Using the naturally available quantum geometry length scale of $\sqrt{q}$, all dimensionfull quantities below, are rendered dimensionless. We will continue to use the same symbols though.

The Hamilton's equations from the effective Hamiltonian are,

$$
\begin{aligned}
\frac{d p^{I}}{d \tau} & =-2 p^{I} \cos \epsilon K_{I}\left(\frac{p^{J} \sin \epsilon K_{J}}{\epsilon}+\frac{p^{K} \sin \epsilon K_{K}}{\epsilon}\right) \\
\frac{d K_{I}}{d \tau} & =+2 \frac{\sin \epsilon K_{I}}{\epsilon}\left(\frac{p^{J} \sin \epsilon K_{J}}{\epsilon}+\frac{p^{K} \sin \epsilon K_{K}}{\epsilon}\right), \\
0 & =\left(\frac{p^{1} \sin \epsilon K_{1}}{\epsilon}\right)\left(\frac{p^{2} \sin \epsilon K_{2}}{\epsilon}\right)+\text { cyclic } .
\end{aligned}
$$

It follows immediately that $\frac{p^{I} \sin \epsilon K_{I}}{\epsilon}:=-\frac{\alpha_{I}}{2}$ are constants of motion, with $\left(\alpha_{1} \alpha_{2}+\right.$ cyclic $)=0 \stackrel{2}{=} \sum_{I} \alpha_{I}^{2}-$ $\left(\sum_{I} \alpha_{I}\right)^{2}$ following from (7).

If all the $\alpha_{I}$ are zero, then all the $p^{I}, K_{I}$ are also constants. Since lapse is non-zero, the $p^{I}$ are non-zero constants and the solution represents the usual Minkowski space-time. The effective dynamics thus retains the Minkowski space-time as a solution indicating a good classical limit of the quantum dynamics. For a non-trivial solution, then, $\sum_{I} \alpha_{I}^{2} \neq 0$ must hold and as usual, by a constant scaling of the $\mathcal{N}$ (or of $\tau$ ), one can arrange the $\alpha_{I}$ to satisfy: $\sum_{I} \alpha_{I}=1$. Thus the the one dimensional parameter space of these solutions is exactly same as that of the usual Kasner solutions. (The special cases of the form $\alpha_{1}=1, \alpha_{2}=\alpha_{3}=0$ are independent of $\epsilon$ in their behaviour and are not considered here. Thus all $\alpha_{I}$ are assumed to be non-zero.) It follows that exactly one of the $\alpha_{I}$ 's must be strictly negative and the remaining two strictly positive and $\alpha_{J}+\alpha_{K}=1-\alpha_{I}>0$.
Since $\alpha_{I}$ are non-zero constants, neither $p^{I}$ nor $\sin \epsilon K_{I}$ can vanish and thus cannot change sign. For definiteness, let us restrict our attention to 'positively oriented' triad and in particular take $p^{I}>0, \forall I$. Then, $\operatorname{sgn}\left(\sin \epsilon K_{I}\right)=$ $-\operatorname{sgn}\left(\alpha_{I}\right)$, which fixes the two quadrants to which the 'angles' $\theta_{I}:=\epsilon K_{I}$ must be confined along a solution i.e. either $0<\epsilon K_{I}<\pi$ or $-\pi<\epsilon K_{I}<0$. Clearly, $p^{I}$ approach $\infty$ as $\epsilon K_{I}$ approach the end points of the intervals and take the minimum value $\frac{\epsilon\left|\alpha_{I}\right|}{2}$ for $\epsilon K_{I}=$ $\pm \frac{\pi}{2}$. The traversal of $\theta_{I}$ with $\tau$ is also fixed by (6): $\theta_{I}$ must decrease if $\sin \theta_{I}$ is positive and increase if $\sin \theta_{I}$ is negative i.e. we must have $\theta_{I} \rightarrow 0_{ \pm}$as $\tau \rightarrow \infty\left(\theta_{I} \rightarrow \pm \pi\right.$ as $\tau \rightarrow-\infty)$.

Eliminating $K_{I}$ in favour of $\alpha_{I}, p^{I}$, leaves us with an equation for $p^{I}$, namely,

$$
\frac{d p^{I}}{d \tau}= \pm\left(1-\alpha_{I}\right) \sqrt{\left(p^{I}\right)^{2}-\left(\frac{\epsilon \alpha_{I}}{2}\right)^{2}} .
$$

The \pm is determined by the quadrant to which the angle $\epsilon K_{I}$ belongs. The solution is easily obtained as,

$$
p^{I}(\tau)=\epsilon \frac{\left|\alpha_{I}\right|}{2} \cosh \left\{\left(1-\alpha_{I}\right)\left(\tau-\left(\tau_{I}\right)_{0}\right)\right\} .
$$

Notice that a triad component attains its smallest value, $\frac{\epsilon\left|\alpha_{I}\right|}{2}$, at $\tau=\left(\tau_{I}\right)_{0}$ while for large $|\tau|$ it behaves as $p^{I} \sim$ $\left(\epsilon\left|\alpha_{I}\right| / 4\right) \exp \left(\left(1-\alpha_{I}\right)|\tau|\right)$.

In terms of the scale factors, $a_{I}=\frac{\text { volume }}{p^{I}}$, the solution is given by,

$$
\begin{aligned}
a_{I}^{2}(\tau)=\epsilon \frac{1-\alpha_{I}}{2}[ & \left(\cosh \left\{\left(1-\alpha_{J}\right)\left(\tau-\left(\tau_{J}\right)_{0}\right)\right\}\right) \times \\
& \left(\cosh \left\{\left(1-\alpha_{K}\right)\left(\tau-\left(\tau_{K}\right)_{0}\right)\right\}\right) \times \\
& \left.\left(\cosh \left\{\left(1-\alpha_{I}\right)\left(\tau-\left(\tau_{I}\right)_{0}\right)\right\}\right)^{-1}\right](10)
\end{aligned}
$$

For comparison, the triad and the scale factor for the Kasner solution are,

$$
p^{I}(\tau)=p_{0}^{I} e^{\left(1-\alpha_{I}\right) \tau} \quad, \quad a_{I}^{2}(\tau)=\left(a_{I}\right)_{0}^{2} e^{2 \alpha_{I} \tau} .
$$

One can recover the Kasner solution from the modified one by taking the limit $\left(\tau_{I}\right)_{0} \rightarrow-\infty, \epsilon \rightarrow 0$ such that $\epsilon \frac{\left|\alpha_{I}\right|}{4} e^{-\left(1-\alpha_{I}\right)\left(\tau_{I}\right)_{0}}=p_{0}^{I}$.

For the Kasner solution, two scale factors vanish and third one diverges such that the volume vanishes exponentially with $\tau$, as $\tau \rightarrow-\infty$. This translates into a finite synchronous time $t$ in the past, making the Kasner solution singular.

By contrast, for the modified solution, none of the triad variables can become zero at any $\tau$ and the volume never vanishes. Furthermore, due to the hyperbolic cosine function, for both asymptotic times, the modified solution approaches the large volume behaviour of the Kasner solution. In particular, the scale factor behaves as $a_{I}^{2} \rightarrow\left(\epsilon\left(1-\alpha_{I}\right) / 4\right) \exp \left(2 \alpha_{I}|\tau|\right)$ as $|\tau| \rightarrow \infty$. Consequently, exactly one scale factor vanishes while the remaining two diverge as $|\tau| \rightarrow \infty$. Thus if one begins with 
a cubical cell at some finite $\tau$, then the cell will become planar after both forward and backward evolution (under Kasner evolution the cell will become planar in 'future' and one dimensional in the 'past)'. Since $\tau \rightarrow \pm \infty$ correspond to $t \rightarrow \pm \infty$, the vanishing/diverging behaviour of scale factors never occurs for finite $t$. The modified solution is thus non-singular.

Consider the behaviour of the volume, $V^{2}=p^{1} p^{2} p^{3}$. For $|\tau| \rightarrow \infty, V^{2} \rightarrow \frac{\epsilon^{3}}{64}\left|\alpha_{1} \alpha_{2} \alpha_{3}\right| e^{2|\tau|}$, and therefore it must have a minimum, $V_{*}$, for some $\tau_{*}$. It is clear that for $\tau$ larger (smaller) than all $\left(\tau_{I}\right)_{0}, V^{2}$ is monotonic. Thus $\tau_{*}$ must lie in the interval of the minimum and maximum values of the parameters $\left(\tau_{I}\right)_{0}$. The minimum volume, $V_{*}$, reached by any particular solution depends on $\vec{\alpha}$ as well as on $\vec{\tau}_{0}$. For any given $\vec{\alpha}$, the smallest possible minimum volume is attained for solutions for which all $\left(\tau_{I}\right)_{0}$ are equal. Parameterizing the $\vec{\alpha}$ as $[1]$ : $\alpha_{1}:=-u /\left(1+u+u^{2}\right), \alpha_{2}:=(1+u) /\left(1+u+u^{2}\right), \alpha_{3}:=(u+$ $\left.u^{2}\right) /\left(1+u+u^{2}\right), u \geq 1$, this smallest possible minimum volume for a given $u$, is given by,

$$
\left(V_{*}\right)_{\min }=\sqrt{\frac{\epsilon^{3}\left|\alpha_{1} \alpha_{2} \alpha_{3}\right|}{8}}=\left(\frac{\epsilon}{2}\right)^{\frac{3}{2}}\left[\frac{u(1+u)}{\left(1+u+u^{2}\right)^{\frac{3}{2}}}\right]
$$

which can be arbitrarily small though strictly positive.

Since singularity theorem is evaded, 'energy' condition(s) must be violated. In the present context, this means that $R_{t t}<0$ must hold some where in the spacetime. By computing $R_{t t}=-\sum_{I} a_{I}^{-1} \frac{d^{2} a_{I}}{d t^{2}}$, one can see easily that this is so in the neighbourhood of $V_{*}$.

So far we have focussed on the features of the exact solution. Recall that for the validity of the effective Hamiltonian we also need $p^{I} \gtrsim q$, or in the dimensionless variables used above, $p^{I} \gtrsim 1$. This puts a restriction on the time $\tau$ for which any specific solution can be trusted as an approximation. Specifically, $\cosh \left\{\left(1-\alpha_{I}\right)\left(\tau-\left(\tau_{I}\right)_{0}\right)\right\} \gtrsim$ $\frac{2}{\epsilon\left|\alpha_{I}\right|}$. Had we used the effective Hamiltonian obtained from the continuum approximation [5, 16] which is same as the Einstein Hamiltonian, we would get the usual Kasner solution and obtain the restriction on $\tau$ as, exp $\left\{\left(1-\alpha_{I}\right)\left(\tau-\left(\tau_{I}\right)_{0}\right)\right\} \gtrsim \frac{4}{\epsilon \mid \alpha_{I}}$. For ease of comparison, we have just written $p_{0}^{I}:=\frac{\epsilon\left|\alpha_{I}\right|}{4} \exp \left\{-\left(1-\alpha_{I}\right)\left(\tau_{I}\right)_{0}\right\}$ so that at for large $\tau$, both the modified and the usual Kasner solutions match. Since the modified solution is always larger than the Kasner solution at the same $\tau$, the $\tau$-regime of validity for the effective solution is larger than that for the Kasner solution. However, since $\frac{2}{\epsilon\left|\alpha_{I}\right|}>\frac{2}{\epsilon}>2$, the parameters $\left(\tau_{I}\right)_{0}$ are out side the domain of validity and so is the smallest (non-zero) value of triad components. The 'bounce' in the triad components is thus in the quantum regime. This is different from the isotropic case [12] where the bounce implied by effective dynamics can occur in the domain of validity of the effective Hamiltonian, depending upon de- tails of matter Hamiltonian. Thus, in the simplest of the anisotropic models, although the effective dynamics inferred is non-singular due to bouncing triad components, these bounces lie in the quantum domain and for a 'reliable' removal of singularity, one still needs to appeal to the quantum theory.

In summary, we make three points: (1) the method of effective Hamiltonian can be extended to homogeneous, anisotropic models and leads to non-singular effective dynamics with the exact solution parameterized by the same Kasner parameters; (2) unlike (at least some of) the isotropic models, for reliable singularity removal, one has to appeal to quantum theory; and (3) the quantum theory stipulates modifications of the classical Einstein dynamics, not only for small triads $\left(p^{I}\right)$ but also for larger values of their conjugates $\left(K_{I}\right)$ which is responsible for the non-singularity.

I would like to thank Martin Bojowald and Golam Hossain for useful remarks. This work was initiated during my visit to AEI, Golm during December 2004. The warm hospitality is gratefully acknowledged.

* Electronic address: shyam@imsc.res.in

[1] V. A. Belinskii, I. M. Khalatnikov, and E. M. Lifschitz, Adv. Phys. 13, (1982) 639.

[2] A. D. Rendall, The nature of space-time singularities, gr-qc/0503112

[3] C. Rovelli, Living Reviews in Relativity 1, (1998) 1; T. Thiemann, Lect. Notes Phys. 631, (2003) 41; A. Ashtekar and J. Lewandowski, Class. Quant. Grav. 21, (2004) R53.

[4] M. Bojowald, Class. Quantum Grav. 20, (2003) 2595.

[5] M. Bojowald, G. Date, and K. Vandersloot, Class. Quantum Grav. 21, (2004) 1253.

[6] M. Bojowald, Class. Quantum Grav. 18, (2001) L109.

[7] G. Date and G. M. Hossain, Class. Quantum Grav., 21, (2004) 4941.

[8] K. Banerjee and G. Date, Class. Quantum Grav, (to appear), 2005.

[9] M. Bojowald, Phys. Rev. D 64, (2001) 084018.

[10] M. Bojowald, Pramana 63, (2004) 765.

[11] M. Bojowald, Phys. Rev. Lett. 89, (2002) 261301; G. Date and G. M. Hossain, Phys. Rev. Lett. 94, (2005) 011301.

[12] G. Date and G. M. Hossain, Phys. Rev. Lett. 94, (2005) 011302.

[13] G. M. Hossain, Primordial Density Perturbation in Effective Loop Quantum Cosmology, gr-qc/0411012

[14] S. Tsujikawa, P. Singh and R. Maartens, Class. Quantum Grav. 21, (2004) 5767.

[15] M. Bojowald and G. Date, Phys. Rev. Lett. 92, (2004) 071302.

[16] M. Bojowald, G. Date, and G. M. Hossain, Class. Quantum Grav. 21, (2004) 3541.

[17] A. Ashtekar, M. Bojowald and J. Lewandowski, Adv. Theor. Math. Phys. 7, (2003) 233. 